\title{
Hybrid methods for accretive variational inequalities involving pseudocontractions in Banach spaces
}

Yaqin Wang ${ }^{1,2^{*}}$ and Rudong Chen ${ }^{3}$

\author{
* Correspondence: \\ wangyaqin0579@126.com \\ 'Department of Mathematics, \\ Shaoxing University, Shaoxing \\ 312000, China \\ Full list of author information is \\ available at the end of the article
}

\begin{abstract}
We use strongly pseudocontractions to regularize a class of accretive variational inequalities in Banach spaces, where the accretive operators are complements of pseudocontractions and the solutions are sought in the set of fixed points of another pseudocontraction. In this paper, we consider an implicit scheme that can be used to find a solution of a class of accretive variational inequalities. Our results improve and generalize some recent results of Yao et al. (Fixed Point Theory Appl, doi:10.1155/2011/180534, 2011) and Lu et al. (Nonlinear Anal, 71(3-4), 1032-1041, 2009).

2000 Mathematics subject classification 47H05; 47H09; 65J15
\end{abstract}

Keywords: hybrid method, accretive operator, variational inequality, pseudocontraction

\section{Introduction}

Throughout this paper, we always assume that $E$ is a real Banach space, $\langle\cdot, \cdot\rangle$ is the dual pair between $E$ and $E^{*}$, and $2^{E}$ denotes the family of all the nonempty subsets of $E$. Let $C$ be a nonempty closed convex subset of $E$ and $T: C \rightarrow E$ be a nonlinear mapping. Denote by $\operatorname{Fix}(T)$ the set of fixed points of $T$, that is, $\operatorname{Fix}(T)=\{x \in C: T x=x\}$. The generalized duality mapping $J: E \rightarrow 2^{E^{*}}$ is defined by

$$
J(x)=\left\{f^{*} \in E^{*}:\left\langle x, f^{*}\right\rangle=\|x\|,\left\|f^{*}\right\|=\|x\|\right\}, \quad \forall x \in E .
$$

In the sequel, we shall denote the single-valued duality mapping by $j$. When $\left\{x_{n}\right\}$ is a sequence in $E, x_{n} \rightarrow x\left(x_{n} \rightarrow x, x_{n} \otimes x\right)$ will denote strong (respectively, weak and weak $\left.^{*}\right)$ convergence of the sequence $\left\{x_{n}\right\}$ to $x$.

A mapping $T$ with domain $D(T)$ and range $R(T)$ in $E$ is called pseudocontractive if the inequality

$$
\|x-y\| \leq\|x-y+t((I-T) x-(I-T) y)\|
$$

holds for each $x, y \in D(T)$ and for all $t>0$. As a result of [1], it follows from (1.1) that $T$ is pseudocontractive if and only if there exists $j(x-y) \in J(x-y)$ such that $\langle T x$ $T y, j(x-y)\rangle \leq\|x-y\|^{2}$ for any $x, y \in D(T)$. T is called strongly pseudocontractive if there exist $j(x-y) \in J(x-y)$ and $\beta \in(0,1)$ such that $\langle T x-T y, j(x-y)\rangle \leq \beta\|x-y\|^{2}$ for any $x, y \in D(T)$. T is called Lipschitzian if there exists $L \geq 0$ such that $\|T x-T y\|$

\section{Springer}


$\leq L|| x-y||, \forall x, y \in D(T)$. If $L=1$, then $T$ is called nonexpansive, and it is called contraction if $L \in[0,1)$.

Let $E=H$ be a Hilbert space with inner product $\langle\cdot, \cdot\rangle$. Recall that $T: C \rightarrow H$ is called monotone if $\langle T x-T y, x-y\rangle \geq 0, \forall x, y \in C$. A variational inequality problem, denoted by $\operatorname{VI}(T, C)$, is to find a point $x^{*}$ with the property

$$
x^{*} \in C \text {, such that }\left\langle T x^{*}, x-x^{*}\right\rangle \geq 0, \quad \forall x \in C .
$$

If the mapping $T$ is a monotone operator, then we say that $\mathrm{VI}(T, C)$ is monotone.

In [2], Lu et al. considered the following type of monotone variational inequality problem in Hilbert spaces(denoted by VI(1.2))

$$
\text { find } x^{*} \in \operatorname{Fix}(T) \text { such that }\left\langle(I-S) x^{*}, x-x^{*}\right\rangle \geq 0, \quad \forall x \in \operatorname{Fix}(T) \text {, }
$$

where $T, S: C \rightarrow C$ are nonexpansive mappings and $\operatorname{Fix}(T) \neq \varnothing$. Let $W$ denote the set of solutions of the $\mathrm{VI}(1.2)$.

Very recently, Yao et al. [3] considered VI(1.2) in Hilbert spaces when $T, S: C \rightarrow C$ are pseudocontractions.

In this paper, we consider the following variational inequality problem in Banach spaces (denoted by VI(1.3))

$$
\text { find } x^{*} \in \operatorname{Fix}(T) \text { such that }\left\langle(I-S) x^{*}, j\left(x-x^{*}\right)\right\rangle \geq 0, \quad \forall x \in \operatorname{Fix}(T),
$$

where $T, S: C \rightarrow C$ are pseudocontractions. Let $\Omega$ denote the set of solutions of the $\mathrm{VI}(1.3)$ and assume that $\Omega$ is nonempty. Since $I-S$ is accretive, then we say $\operatorname{VI}(1.3)$ is an accretive variational inequality.

For solving the $\operatorname{VI}(T, C)$, hybrid methods were studied by Yamada [4] where he assumed that $T$ is Lipschitzian and strongly monotone. However, his methods do not apply to the VI(1.2) since the mapping $I-S$ fails, in general, to be strongly monotone, though it is Lipschitzian. In fact the $\mathrm{VI}(1.2)$ is, in general, ill-posed, and thus regularization is needed. Let $T, S: C \rightarrow C$ be nonexpansive and $f: C \rightarrow C$ be contractive. In 2006, Moudafi and Mainge [5] studied the VI(1.2) by regularizing the mapping $t S+(1$ $-t) T$ and defined $\left\{x_{s, t}\right\}$ as follows:

$$
x_{s, t}=s f\left(x_{s, t}\right)+(1-s)\left[t S x_{s, t}+(1-t) T x_{s, t}\right], \quad s, t \in(0,1) .
$$

Since Moudafi and Mainge's regularization depends on $t$, the convergence of the scheme (1.4) is more complicated. So Lu et al. [2] defined $\left\{x_{s, t}\right\}$ as follows by regularizing the mapping $S$ :

$$
x_{s, t}=s\left[t f\left(x_{s, t}\right)+(1-t) S x_{s, t}\right]+(1-s) T x_{s, t}, \quad s, t \in(0,1) .
$$

Note that Lu et al.'s regularization does no longer depend on $t$. And their result for the regularization (1.5) is under dramatically less restrictive conditions than Moudafi and Mainge's [5].

Very recently, Yao et al. [3] extended Lu et al.'s result to a general case, i.e., in the scheme (1.5), $S, T$ are extended to Lipschitz pseudocontractive and $f$ is extended to strongly pseudocontractive. But in [3], after careful discussion, we observe that a continuity condition on $f$ is necessary. So, in this paper, we modify it.

Motivated and inspired by the above work, in this paper, we use strongly pseudocontrations to regularize the ill-posed accretive $\mathrm{VI}(1.3)$, and analyze the convergence of 
the scheme (1.5). The results we obtained improve and extend the corresponding results in $[2,3]$.

\section{Preliminaries}

If Banach space $E$ admits sequentially continuous duality mapping $J$ from weak topology from weak" topology, then by [6, Lemma 1$]$, we get that duality mapping $J$ is single-valued. In this case, duality mapping $J$ is also said to be weakly sequentially continuous, i.e., for each $\left\{x_{n}\right\} \subset E$ with $x_{n} \rightarrow x$, then $J\left(x_{n}\right) \otimes J x[6,7]$.

A Banach space $E$ is said to be satisfying Opial's condition if for any sequence $\left\{x_{n}\right\}$ in $E, x_{n} \rightarrow x(n \rightarrow \infty)$ implies that

$$
\limsup _{n \rightarrow \infty}\left\|x_{n}-x\right\|<\limsup _{n \rightarrow \infty}\left\|x_{n}-y\right\|, \quad \forall y \in E, \text { with } y \neq x .
$$

By [6, Lemma 1], we know that if $E$ admits a weakly sequentially continuous duality mapping, then $E$ satisfies Opial's condition.

Lemma 2.1([7]) Let $C$ be a nonempty closed convex subset of a reflexive Banach space $E$, which satisfies Opial's condition, and suppose $T: C \rightarrow E$ is nonexpansive. Then, the mapping $I-T$ is demiclosed at zero, i.e.,

$$
x_{n} \rightarrow x, \quad x_{n}-T x_{n} \rightarrow 0 \text { implies } x=T x .
$$

Recall that $S: C \rightarrow C$ is called accretive if $I-S$ is pseudocontractive. We denote by $J_{r}$ the resolvent of $S$, i.e., $J_{r}=(I+r S)^{-1}$. It is well known that $J_{r}$ is nonexpansive, singlevalued and $\operatorname{Fix}\left(J_{r}\right)=S^{-1}(0)=\{z \in D(S): 0=S z\}$ for all $r>0$. For more details, see [8-10].

Let $T: C \rightarrow C$ be a pseudocontractive mapping; then, $I-T$ is accretive. We denote $A$ $=J_{1}=(2 I-T)^{-1}$. Then, Fix $(A)=F i x(T)$ and $A: R(2 I-T) \rightarrow K$ is nonexpansive and single-valued. The following lemma can be found in [11].

Lemma 2.2([11]) Let $C$ be a nonempty closed convex subset of a real Banach space $E$ and $T: C \rightarrow C$ be a continuous pseudocontractive map. We denote $A=J_{1}=(2 I-$ $T)^{-1}$. Then,

(i) [12, Theorem 6] The map $A$ is a nonexpansive self-mapping on $C$, i.e., for all $x$, $y \in C$, there hold

$$
\|A x-A y\| \leq\|x-y\| \text { and } A x \in C ;
$$

(ii) If $\lim _{n \rightarrow \infty}|| x_{n}-T x_{n} \|=0$, then $\lim _{n \rightarrow \infty}|| x_{n}-A x_{n} \|=0$.

We also need the following lemma.

Lemma 2.3 Let $C$ be a nonempty closed convex subset of a real Banach space $E$. Assume that $F: C \rightarrow E$ is accretive and weakly continuous along segments; that is $F(x$ $+t y) \rightarrow F(x)$ as $t \rightarrow 0$. Then, the variational inequality

$$
x^{*} \in C, \quad\left\langle F x^{*}, j\left(x-x^{*}\right)\right\rangle \geq 0, \quad \forall x \in C
$$


is equivalent to the dual variational inequality

$$
x^{*} \in C, \quad\left\langle F x, j\left(x-x^{*}\right)\right\rangle \geq 0, \quad \forall x \in C .
$$

Proof $(2.1) \Rightarrow(2.2)$ Since $F$ is accretive, we have

$$
\left\langle F x-F x^{*}, j\left(x-x^{*}\right)\right\rangle \geq 0
$$

and so

$$
\left\langle F x, j\left(x-x^{*}\right)\right\rangle \geq\left\langle F x^{*}, j\left(x-x^{*}\right)\right\rangle \geq 0 .
$$

(2.2) $\Rightarrow(2.1)$ For any $x \in C$, put $w=t x+(1-t) x^{*}, \forall t \in(0,1)$. Then, $w \in C$. Taking $x=w$ in (2.2), we have

$$
\left\langle F w, j\left(w-x^{*}\right)\right\rangle=t\left\langle F w, j\left(x-x^{*}\right)\right\rangle \geq 0,
$$

i.e.,

$$
\left\langle F w, j\left(x-x^{*}\right)\right\rangle \geq 0 .
$$

Letting $t \rightarrow 0$ in the above inequality, since $F$ is weakly continuous along segments, it follows that (2.1) holds.

\section{Main Results}

Let $C$ be a nonempty closed convex subset of a real Banach space $E$. Let $f: C \rightarrow C$ be a Lipschitz strongly pseudocontraction and $T, S: C \rightarrow C$ be two continuous pseudocontractions. For $s, t \in(0,1)$, we define the following mapping

$$
x \mapsto W_{s, t} x:=s[t f(x)+(1-t) S x]+(1-s) T x .
$$

It is easy to see that the mapping $W_{s, t}: C \rightarrow C$ is a continuous strongly pseudocontractive mapping. So, by [13], $W_{s, t}$ has a unique fixed point which is denoted $x_{s, t} \in C$; that is

$$
x_{s, t}=W_{s, t} x_{s, t}=s\left[t f\left(x_{s, t}\right)+(1-t) S x_{s, t}\right]+(1-s) T x_{s, t}, \quad s, t \in(0,1) .
$$

Theorem 3.1 Let $E$ be a reflexive Banach space that admits a weakly sequentially continuous duality mapping from $E$ to $E^{*}$. Let $C$ be a nonempty closed convex subset of $E$. Let $f: C \rightarrow C$ be a Lipschitz strongly pseudocontraction, $S: C \rightarrow C$ be a Lipschitz pseudocontraction, and $T: C \rightarrow C$ be a continuous pseudocontraction with $\operatorname{Fix}(T) \neq$ $\varnothing$. Suppose that the solution set $\Omega$ of the $\operatorname{VI}(1.3)$ is nonempty. Let for each $(s, t) \in(0$, $1)^{2},\left\{x_{s, t}\right\}$ be defined by (3.1). Then, for each fixed $t \in(0,1)$, the net $\left\{x_{s, t}\right\}$ converges in norm, as $s \rightarrow 0$, to a point $x_{t} \in \operatorname{Fix}(T)$. Moreover, as $t \rightarrow 0$, the net $\left\{x_{t}\right\}$ converges in norm to the unique solution $x^{*}$ of the following inequality variational(denoted by VI (3.2)):

$$
x^{*} \in \Omega,\left\langle(I-f) x^{*}, J\left(x-x^{*}\right)\right\rangle \geq 0, \quad \forall x \in \Omega .
$$

Hence, for each null sequence $\left\{t_{n}\right\}$ in $(0,1)$, there exists another null sequence $\left\{s_{n}\right\}$ in $(0,1)$, such that the sequence $x_{s_{n}, t_{n}} \rightarrow x^{*}$ in norm as $n \rightarrow \infty$.

Proof We divide our proofs into several steps as follows.

Step 1 For each fixed $t \in(0,1)$, the net $\left\{x_{s, t}\right\}$ is bounded. 
For any $z \in \operatorname{Fix}(T)$, for all $s, t \in(0,1)$, by (3.1), we have

$$
\begin{aligned}
\left\|x_{s, t}-z\right\|^{2}= & \left\langle s\left[t f\left(x_{s, t}\right)+(1-t) S x_{s, t}\right]+(1-s) T x_{s, t}-z, J\left(x_{s, t}-z\right)\right\rangle \\
= & s t\left\langle f\left(x_{s, t}\right)-f(z), J\left(x_{s, t}-z\right)\right\rangle+s(1-t)\left\langle S x_{s, t}-S z, J\left(x_{s, t}-z\right)\right\rangle \\
& +(1-s)\left\langle T x_{s, t}-T z, J\left(x_{s, t}-z\right)\right\rangle+s t\left\langle f(z)-z_{,} J\left(x_{s, t}-z\right)\right\rangle \\
& +s(1-t)\left\langle S z-z_{,} J\left(x_{s, t}-z\right)\right\rangle \\
\leq & s t \beta\left\|x_{s, t}-z\right\|^{2}+s(1-t)\left\|x_{s, t}-z\right\|^{2}+(1-s)\left\|x_{s, t}-z\right\|^{2} \\
& +s t\|f(z)-z\|\left\|x_{s, t}-z\right\|+s(1-t)\|S z-z\|\left\|x_{s, t}-z\right\| \\
= & (1-s t(1-\beta))\left\|x_{s, t}-z\right\|^{2}+s[t|| f(z)-z \| \\
& +(1-t)\|S z-z\|]\left\|x_{s, t}-z\right\|,
\end{aligned}
$$

which implies that

$$
\begin{aligned}
\left\|x_{s, t}-z\right\| & \leq \frac{t|| f(z)-z \|}{t(1-\beta)}+\frac{(1-t)\|S z-z\|}{t(1-\beta)} \\
& \leq \frac{1}{t(1-\beta)} \max \{\|f(z)-z\|,\|S z-z\|\} .
\end{aligned}
$$

Hence, for each $t \in(0,1),\left\{x_{s, t}\right\}$ is bounded. Furthermore, by the Lipschitz continuity of $f$ and $S$, we obtain $\left\{f\left(x_{s, t}\right)\right\}$ and $\left\{S x_{s, t}\right\}$, which are both bounded for each $t \in(0,1)$. From (3.1), we have

$$
\left\|T x_{s, t}\right\| \leq \frac{1}{1-s}\left\|x_{s, t}\right\|+\frac{s}{1-s}\left\|t f\left(x_{s, t}\right)+(1-t) S x_{s, t}\right\| .
$$

So $\left\{T x_{s, t}\right\}$ is also bounded as $s \rightarrow 0$ for each $t \in(0,1)$.

Step $2 x_{s, t} \rightarrow x_{t} \in \operatorname{Fix}(T)$ as $s \rightarrow 0$.

From (3.1), for each $t \in(0,1)$, we get

$$
x_{s, t}-T x_{s, t}=s\left[t f\left(x_{s, t}\right)+(1-t) S x_{s, t}-T x_{s, t}\right] \rightarrow 0, \quad \text { as } s \rightarrow 0 .
$$

It follows from (3.1) that

$$
\begin{aligned}
\left\|x_{s, t}-z\right\|^{2}= & s t\left\langle f\left(x_{s, t}\right)-f(z), J\left(x_{s, t}-z\right)\right\rangle+s(1-t)\left\langle S x_{s, t}-S z, J\left(x_{s, t}-z\right)\right\rangle \\
& +(1-s)\left\langle T x_{s, t}-T z, J\left(x_{s, t}-z\right)\right\rangle+s t\left\langle f(z)-z, J\left(x_{s, t}-z\right)\right\rangle \\
& +s(1-t)\left\langle S z-z, J\left(x_{s, t}-z\right)\right\rangle \\
\leq & (1-s t(1-\beta))\left\|x_{s, t}-z\right\|^{2}+s t\left\langle f(z)-z, J\left(x_{s, t}-z\right)\right\rangle \\
& +s(1-t)\left\langle S z-z_{,} J\left(x_{s, t}-z\right)\right\rangle .
\end{aligned}
$$

It turns out that

$$
\left\|x_{s, t}-z\right\|^{2} \leq \frac{1}{t(1-\beta)}\left\langle t f(z)+(1-t) S z-z, J\left(x_{s, t}-z\right)\right\rangle, \quad \forall z \in \operatorname{Fix}(T) .
$$

Assume that $\left\{s_{n}\right\} \subset(0,1)$ is such that $s_{n} \rightarrow 0(n \rightarrow \infty)$, by the above inequality we have

$$
\left\|x_{s_{n}, t}-z\right\|^{2} \leq \frac{1}{t(1-\beta)}\left\langle t f(z)+(1-t) S z-z J\left(x_{s_{n, t}}-z\right)\right\rangle, \quad \forall z \in \operatorname{Fix}(T) .
$$

Since $\left\{x_{s_{n}, t}\right\}$ is bounded, without loss of generality, we may assume that as $s_{n} \rightarrow 0$, $x_{s_{n}, t} \rightarrow x_{t}$. Combining (3.3), Lemma 2.1 and 2.2, we obtain $x_{t} \in \operatorname{Fix}(A)=\operatorname{Fix}(T)$. Taking $z=x_{t}$ in (3.4), we get 


$$
\left\|x_{s_{n}, t}-x_{t}\right\|^{2} \leq \frac{1}{t(1-\beta)}\left\langle t f\left(x_{t}\right)+(1-t) S x_{t}-x_{t}, \quad J\left(x_{s_{n}, t}-x_{t}\right)\right\rangle .
$$

Since $x_{s_{n}, t} \rightarrow x_{t}$ and $J$ is weakly sequentially continuous, by (3.5) as $s_{n} \rightarrow 0$, we obtain $x_{s_{n}, t} \rightarrow x_{t}$. This has proved that the relative norm compactness of the net $\left\{x_{s, t}\right\}$ as $s \rightarrow$ 0 .

Letting $n \rightarrow \infty$ in (3.4), we obtain

$$
\left\|x_{t}-z\right\|^{2} \leq \frac{1}{t(1-\beta)}\left\langle t f(z)+(1-t) S z-z_{1} J\left(x_{t}-z\right)\right\rangle, \quad \forall z \in \operatorname{Fix}(T) .
$$

So, $x_{t}$ is a solution of the following variational inequality:

$$
x_{t} \in \operatorname{Fix}(T),\left\langle t f(z)+(1-t) S z-z_{1} J\left(x_{t}-z\right)\right\rangle \geq 0, \quad \forall z \in \operatorname{Fix}(T) .
$$

Letting $C=F i x(T), F=t(I-f)+(1-t)(I-S)$, by Lemma 2.3, we have the equivalent dual variational inequality:

$$
x_{t} \in \operatorname{Fix}(T),\left\langle t f\left(x_{t}\right)+(1-t) S x_{t}-x_{t}, J\left(x_{t}-z\right)\right\rangle \geq 0, \quad \forall z \in \operatorname{Fix}(T) .
$$

Next, we prove that for each $t \in(0,1)$, as $s \rightarrow 0,\left\{x_{s, t}\right\}$ converges in norm to $x_{t} \in$ Fix $(T)$. Assume $x_{s^{\prime}, t} \rightarrow x_{t}^{\prime}$ as $s_{n}^{\prime} \rightarrow 0$. Similar to the above proof, we have $x_{t}^{\prime} \in \operatorname{Fix}(T)$, which solves the following variational inequality:

$$
x_{t}^{\prime} \in \operatorname{Fix}(T),\left\langle t f\left(x_{t}^{\prime}\right)+(1-t) S x_{t}^{\prime}-x_{t}^{\prime} J\left(x_{t}^{\prime}-z\right)\right\rangle \geq 0, \quad \forall z \in \operatorname{Fix}(T) .
$$

Taking $z=x_{t}^{\prime}$ in (3.7) and $z=x_{t}$ in (3.8), we have

$$
\begin{aligned}
& \left\langle t f\left(x_{t}\right)+(1-t) S x_{t}-x_{t}, J\left(x_{t}-x_{t}^{\prime}\right)\right\rangle \geq 0, \\
& \left\langle t f\left(x_{t}^{\prime}\right)+(1-t) S x_{t}^{\prime}-x_{t}^{\prime} J\left(x_{t}^{\prime}-x_{t}\right)\right\rangle \geq 0 .
\end{aligned}
$$

Adding up (3.9) and (3.10), and since $f$ is strongly pseudocontractive and $S$ is pseudocontractive, we have

$$
\begin{aligned}
0 & \leq t\left\langle(I-f) x_{t}-(I-f) x^{\prime}{ }_{t} J\left(x^{\prime}{ }_{t}-x_{t}\right)\right\rangle+(1-t)\left\langle(I-S) x_{t}-(I-S) x^{\prime}{ }_{t} J\left(x^{\prime}{ }_{t}-x_{t}\right)\right\rangle \\
& \leq-t(1-\beta)\left\|x^{\prime}{ }_{t}-x_{t}\right\|^{2},
\end{aligned}
$$

which implies that $x_{t}^{\prime}=x_{t}$. Hence, the net $\left\{x_{s, t}\right\}$ converges in norm to $x_{t} \in \operatorname{Fix}(T)$ as $s$ $\rightarrow 0$.

Step $3\left\{x_{t}\right\}$ is bounded.

Since $\Omega \subset \operatorname{Fix}(T)$, for any $y \in \Omega$, taking $z=y$ in (3.7) we obtain

$$
\left\langle t f\left(x_{t}\right)+(1-t) S x_{t}-x_{t}, J\left(x_{t}-y\right)\right\rangle \geq 0 .
$$

Since $I-S$ is accretive, for any $y \in \Omega$, we have

$$
\left\langle S x_{t}-x_{t}, J\left(x_{t}-y\right)\right\rangle \leq\left\langle S y-y_{,} J\left(x_{t}-y\right)\right\rangle \leq 0 .
$$

Combining (3.11) and (3.12), we have

$$
\left\langle f\left(x_{t}\right)-x_{t}, J\left(x_{t}-y\right)\right\rangle \geq 0, \quad \forall y \in \Omega,
$$

i.e.,

$$
\left\langle f\left(x_{t}\right)-y+y-x_{t}, J\left(x_{t}-y\right)\right\rangle \geq 0, \quad \forall y \in \Omega .
$$


Hence,

$$
\begin{aligned}
\left\|x_{t}-y\right\|^{2} & \leq\left\langle f\left(x_{t}\right)-y_{1} J\left(x_{t}-y\right)\right\rangle \\
& =\left\langle f\left(x_{t}\right)-f(y), J\left(x_{t}-y\right)\right\rangle+\left\langle f(y)-y_{1} J\left(x_{t}-y\right)\right\rangle \\
& \leq \beta\left\|x_{t}-y\right\|^{2}+\left\langle f(y)-y_{1} J\left(x_{t}-y\right)\right\rangle .
\end{aligned}
$$

Hence,

$$
\left\|x_{t}-y\right\|^{2} \leq \frac{1}{1-\beta}\left\langle f(\gamma)-\gamma, \quad J\left(x_{t}-y\right)\right\rangle,
$$

which implies that

$$
\left\|x_{t}-y\right\| \leq \frac{1}{1-\beta}\|f(\gamma)-y\| .
$$

So $\left\{x_{t}\right\}$ is bounded.

Step $4 x_{t} \rightarrow x^{*} \in \Omega$ which is a solution of variational inequality (3.2).

Since $f$ is strongly pseudocontractive, it is easy to see that the solution of the variational inequality (3.2) is unique.

Next, we prove that $\omega_{w}\left(x_{t}\right) \subset \Omega$; namely, if $\left(t_{n}\right)$ is a null sequence in $(0,1)$ such that $x_{t_{n}} \rightarrow x^{\prime}$ as $n \rightarrow \infty$, then $x^{\prime} \in \Omega$. Indeed, it follows from (3.7) that

$$
\left\langle(I-S) x_{t}, J\left(z-x_{t}\right)\right\rangle \geq \frac{t}{1-t}\left\langle(I-f) x_{t}, \quad J\left(x_{t}-z\right)\right\rangle .
$$

Since $I-S$ is accretive, from the above inequality, we have

$$
\left\langle(I-S) z, J\left(z-x_{t}\right)\right\rangle \geq \frac{t}{1-t}\left\langle(I-f) x_{t}, J\left(x_{t}-z\right)\right\rangle, \quad \forall z \in \operatorname{Fix}(T) .
$$

Letting $t=t_{n} \rightarrow 0$ in (3.15), we have

$$
\left\langle(I-S) z, J\left(z-x^{\prime}\right)\right\rangle \geq 0, \quad \forall z \in \operatorname{Fix}(T),
$$

which is equivalent to its dual variational inequality by Lemma 2.3

$$
\left\langle(I-S) x^{\prime}, J\left(z-x^{\prime}\right)\right\rangle \geq 0, \quad \forall z \in \operatorname{Fix}(T) .
$$

Since $\operatorname{Fix}(T)$ is closed convex, then $\operatorname{Fix}(T)$ is weakly closed. Thus, $x^{\prime} \in \operatorname{Fix}(T)$ by virtue of $x_{t} \in \operatorname{Fix}(T)$. So, $x^{\prime} \in \Omega$.

Finally, we show that $x^{\prime}=x^{*}$, the unique solution of $\mathrm{VI}(3.2)$. In fact, taking $t=t_{n}$ and $y=x^{\prime}$ in (3.14), we obtain

$$
\left\|x_{t_{n}}-x^{\prime}\right\|^{2} \leq \frac{1}{1-\beta}\left\langle f\left(x^{\prime}\right)-x^{\prime}, \quad J\left(x_{t_{n}}-x^{\prime}\right)\right\rangle,
$$

which together with $x_{t_{n}} \rightarrow x^{\prime}$ implies that $x_{t_{n}} \rightarrow x^{\prime}$ as $t_{n} \rightarrow 0$. Let $t=t_{n} \rightarrow 0$ in (3.13), we have

$$
\left\langle f\left(x^{\prime}\right)-x^{\prime}, J\left(x^{\prime}-y\right)\right\rangle \geq 0, \quad \forall y \in \Omega .
$$

It follows from (3.16) and $x^{\prime} \in \Omega$ that $x^{\prime}$ is a solution of $\mathrm{VI}(3.2)$. By uniqueness, we have $x^{\prime}=x^{*}$. Therefore, $x_{t} \rightarrow x^{*}$ as $t \rightarrow 0$.

By Theorem 3.1, we have the following corollary directly. 
Corollary 3.1([2, Theorem 3.3]) Let $C$ be a nonempty closed convex subset of a real Hilbert space $H$. Let $f: C \rightarrow C$ be a contraction, $S, T: C \rightarrow C$ be nonexpansive with $\operatorname{Fix}(T) \neq \varnothing$. Suppose that the solution set $W$ of the VI(1.2) is nonempty. Let for each $(s, t) \in(0,1)^{2},\left\{x_{s, t}\right\}$ be defined by (3.1). Then, for each fixed $t \in(0,1)$, the net $\left\{x_{s, t}\right\}$ converges in norm, as $s \rightarrow 0$, to a point $x_{t} \in \operatorname{Fix}(T)$. Moreover, as $t \rightarrow 0$, the net $\left\{x_{t}\right\}$ converges in norm to the unique solution $x^{*}$ of the following inequality variational:

$$
x^{*} \in W,\left\langle(I-f) x^{*}, x-x^{*}\right\rangle \geq 0, \quad \forall x \in W .
$$

Hence, for each null sequence $\left\{t_{n}\right\}$ in $(0,1)$, there exists another null sequence $\left\{s_{n}\right\}$ in $(0,1)$, such that the sequence $x_{s_{n}, t_{n}} \rightarrow x^{*}$ in norm as $n \rightarrow \infty$.

Remark Theorem 3.1 improves and generalizes Theorem 3.1 of Yao et al.[3] in the following aspects:

(i) Theorem 3.1 generalizes Theorem 3.1 in [3] from Hilbert spaces to more general Banach spaces;

(ii) The mappings $T$ in [3, Theorem 3.1] is weakened from Lipschitzian to continuous;

(iii) We modify the condition of $f$, i.e., we suppose that $f$ is Lipschitz strongly pseudocontractive.

The authors declare that they have no competing interests.

\section{Acknowledgements}

The first author was supported by the Research Project of Shaoxing University(No. 09LG1002), and the second author was supported partly by NSFC Grants(No.11071279).

\section{Author details}

${ }^{1}$ Department of Mathematics, Shaoxing University, Shaoxing 312000, China ${ }^{2}$ Mathematical College, Sichuan University, Chengdu, Sichuan 610064, China ${ }^{3}$ Department of Mathematics, Tianjin Polytechnic University, Tianjin 300160, China

\section{Authors' contributions}

The authors discussed the idea of this manuscript. YW drafted the manuscript and RC helped to complete it. We discussed some details by Email. All authors read and approved the final manuscript.

Received: 3 June 2011 Accepted: 8 October 2011 Published: 8 October 2011

\section{References}

1. Kato, T: Nonlinear semi-groups and evolution equations. J Math Soc Jpn. 19(4):508-520 (1967). doi:10.2969/jmsj/ 01940508

2. Lu, XW, Xu, HK, Yin, XM: Hybrid methods for a class of monotone variational inequalities. Nonlinear Anal. 71(34):1032-1041 (2009). doi:10.1016/j.na.2008.11.067

3. Yao, YH, Marino, G, Liou, YC: A hybrid method for monotone variational inequalities involving pseudocontractions. Fixed Point Theory Appl. (2011)

4. Yamada, I: The hybrid steepest descent for the variational inequality problems over the intersection of fixed point sets of nonexpansive mappings, in: Inherently Parallel Algorithms in Feasibility and Optimization and Their Applications. Elsevier, New York. 473-504 (2001)

5. Moudafi, A, Mainge, PE: Towards viscosity approximations of hierarchical fixed-point problems. Fixed Point Theory Appl. 2006(1):1-10 (2006)

6. Gossez, JP, LamiDozo, E: Some geometric properties related to the fixed point theory for nonexpansive mappings. Pac J Math. 40(3), 565-573 (1972)

7. Jung, JS: Iterative approaches to common fixed points of nonexpansive mappings in Banach spaces. J Math Anal Appl. 302(2):509-520 (2005). doi:10.1016/j.jmaa.2004.08.022

8. Reich, S: An iterative procedure for constructing zeros of accretive sets in Banach spaces. Nonlinear Anal. 2(1), 85-92 (1978). doi:10.1016/0362-546X(78)90044-5

9. Takahashi, W: Nonlinear Functional Analysis: Fixed Point Theory and its Applications. Yokohama Publishers Inc., Yokohama (2002)

10. Deimling, K: Zero of accretive operators. Manuscr Math. 13(4), 365-374 (1974). doi:10.1007/BF01171148

11. Song, YS, Chen, RD: Convergence theorems of iterative algorithms for continuous pseudocontractive mappings. Nonlinear Anal. 67(2), 486-497 (2007). doi:10.1016/j.na.2006.06.009 
12. Megginson, RE: An Introduction to Banach Space Theory. Springer, New York (1998)

13. Chang, SS, Cho, YJ, Zhou, HY: Iterative Methods for Nonlinear Operator Equations in Banach Spaces. pp. 12-13. Nova Science Publishers, Inc., Huntington, New York (2002)

doi:10.1186/1687-1812-2011-63

Cite this article as: Wang and Chen: Hybrid methods for accretive variational inequalities involving

pseudocontractions in Banach spaces. Fixed Point Theory and Applications 2011 2011:63.

Submit your manuscript to a SpringerOpen ${ }^{\odot}$ journal and benefit from:

- Convenient online submission

- Rigorous peer review

- Immediate publication on acceptance

- Open access: articles freely available online

- High visibility within the field

- Retaining the copyright to your article

Submit your next manuscript at $\boldsymbol{\nabla}$ springeropen.com 